\title{
Limit shape of Jarník's polygonal curve is a circle
}

\author{
by \\ JovišA Žunić (Cardiff and Belgrade)
}

1. Introduction. In this paper we consider a class of convex lattice polygons which have the minimum possible Euclidean perimeter with respect to their number of vertices. In other words, if $\operatorname{per}(P)$ denotes the Euclidean perimeter of $P$, and $p(n)$ is defined as

$$
p(n)=\min \{\operatorname{per}(P): P \text { is a convex lattice } n \text {-gon }\}
$$

then a convex lattice $n$-gon $P$ is said to be optimal if its perimeter is equal to $p(n)$. Such a polygon will be denoted by $P_{n}$, i.e., $\operatorname{per}\left(P_{n}\right)=p(n)$.

Jarník introduced a special subsequence $P_{n(t)}$ of the (previously defined) optimal polygons for a different reason. He was looking for a strictly convex curve of length at most $s$ containing the maximum number $f(s)$ of points of $\mathbb{Z}^{2}$ and his main aim was to find the magnitude of $f(s)$ when $s$ tends to infinity. He shown that if $G$ is a strictly convex curve of a length $s$, then the maximum number of integer points lying on $G$ is equal to

$$
\frac{3}{\sqrt[3]{2 \pi}} s^{2 / 3}+\mathcal{O}\left(s^{1 / 3}\right)
$$

The exponent and constant are best possible.

In this paper our attention is focused on convex lattice polygons and not on strictly convex curves passing through their vertices. Our purpose is to show the existence and to describe the limit shape of an arbitrary sequence of optimal polygons $P_{n}$ when $n \rightarrow \infty$. Precisely, we show that after the normalization applied to an arbitrary sequence of optimal convex lattice polygons, $P_{n}, n=3,4, \ldots$, the resulting sequence of normalized convex $n$-gons $P_{n}^{\prime}$ tends to a circle as $n \rightarrow \infty$. The normalization is made with respect to the diameter taken in the sense of the $l_{\infty}$-metric-i.e., if a shape $S$ is given and $\Delta(S)$ denotes the diameter of $S$ taken in the sense of the $l_{\infty}$-metric then the normalized shape, denoted by $S^{\prime}$, is obtained by

2000 Mathematics Subject Classification: 11H06, $52 \mathrm{C} 05$.

Key words and phrases: integer geometry, combinatorial optimization, convex lattice polygons. 
replacing every point $(x, y)$ of $S$ by $(x / \Delta(S), y / \Delta(S))$. In other words, we set $S^{\prime}=\Delta(S)^{-1} \cdot S$. In order to obtain an equation of the limit shape of the sequences of optimal convex lattice polygons, we consider the coordinates of vertices of normalized optimal polygons $P_{n}^{\prime}$, given in a parametric form $(\widetilde{x}(n, \alpha), \widetilde{y}(n, \alpha))$ ( $\alpha$ is a suitably chosen parameter), and show that the limits

$$
x=x(\alpha)=\lim _{n \rightarrow \infty} \widetilde{x}(n, \alpha) \quad \text { and } \quad y=y(\alpha)=\lim _{n \rightarrow \infty} \widetilde{y}(n, \alpha)
$$

exist independently of which sequence of optimal $n$-gons is taken $\left(P_{n}\right.$ and $P_{n}^{\prime}$ are uniquely determined only for certain values of $\left.n\right)$. It will turn out that those limits define a circle.

Roughly speaking, here we consider a "pointwise limit shape" of a sequence $P_{n}$ when $n \rightarrow \infty$. Other types of limit shape theorems have been considered recently. Vershik has proved (see [9]) that almost all convex $n^{-1} \cdot \mathbb{Z}^{2}$ lattice polygons lying in the square $[-1,1]^{2}$ are very close to a fixed curve as $n \rightarrow \infty$. This curve is $\sqrt{|y|}+\sqrt{|x|}=1$. Such a limit shape is of a statistical nature - no sequence tending to the limit curve was pointed out. The central limit theorem in the case of this statistical interpretation of the limit shape is proved in [7]. We also refer to a more recent paper [2] which contains an extension of the result from [9] (relating to $[-1,1]^{2}$ ) to every compact planar set $\mathbf{K}$ with a nonempty interior - in addition it characterizes the limit shape as the convex curve with the maximum affine perimeter among all convex curves contained in $\mathbf{K}$.

Let us mention here a few related results. In [8] it is shown that the exponent $2 / 3$ (in Jarník's result) can be decreased by imposing suitable smoothness conditions on $G$. In particular, if $G$ has a continuous third derivative with a sensible bound, the best possible value of the exponent lies between $3 / 5$ and $1 / 3$ inclusive. The generalization of this result to higher dimensions is given in [6]. Also (see [3]), if $G$ is the graph of the function $f$ then the assumptions $f \in C^{d}([0, N]),|f| \leq N,\left|f^{\prime}\right| \leq 1, f^{d} \neq 0$ in $[0, N]$ imply $\left|G \cap \mathbb{Z}^{2}\right| \leq c\left(\varepsilon_{d}\right) N^{1 / 2+\varepsilon_{d}}$ where $\varepsilon_{d} \rightarrow 0$ as $d \rightarrow \infty$. In particular, if $f \in C^{\infty}([0,1])$ is strictly convex then $\left|t \cdot G \cap \mathbb{Z}^{2}\right| \leq c(f, \varepsilon) t^{1 / 2+\varepsilon}$ for every $\varepsilon>0$. In view of the example $f(x)=\sqrt{x}$ the exponent $1 / 2$ is best possible.

We continue with the necessary definitions and notations (Section 2) and derive the limit shape theorem (Section 3 ) for a subsequence of the optimal convex lattice polygons having $n(t)=4 \sum_{s=1}^{t} U(s), t=1,2, \ldots$, vertices, where $U(s)$ is a partition function. The extension from Jarník's sequence $P_{n(t)}$ to the whole class of optimal convex lattice polygons $P_{n}, n=3,4, \ldots$, is straightforward.

2. Preliminaries. A convex lattice polygon is a polygon whose vertices all have integer coordinates and whose interior angles are all strictly less 
than $\pi$ radians (no three vertices are collinear). A polygon with $n$ vertices will be called an $n$-gon.

The dilation of a set $S$ by a given factor $r$ is denoted by $r \cdot S$.

If $a$ and $b$ are integers, then $a \perp b$ means that $a$ and $b$ are relatively prime (i.e., $\operatorname{gcd}(a, b)=1)$.

$U(n)$ is the partition function which counts the number of all positive solutions of $n=q^{2}+p^{2}$ where $q$ and $p$ are relatively prime integers and the order of the numbers is taken into account. For example, $U(170)=4$, because of $1^{2}+13^{2}=13^{2}+1^{2}=7^{2}+11^{2}=11^{2}+7^{2}=170 . U(1)$ is defined to be 1 .

$\mu(n)$ is the Möbius function $([1])$.

For an edge $e=\left[\left(x_{1}, y_{1}\right),\left(x_{2}, y_{2}\right)\right]$ the differences $\left|x_{2}-x_{1}\right|$ and $\left|y_{2}-y_{1}\right|$ are denoted by $x(e)$ and $y(e)$, respectively. For practical reasons, we define the slope of $e$ as $y(e) / x(e)$. For a given integer $t$ we define the set $S(t)$ in the following way:

$$
S(t)=\left\{p / q: p^{2}+q^{2} \leq t, p \perp q, p \text { and } q \text { are positive integers }\right\} .
$$

The Euclidean norm of a point $\mathbf{x} \in \mathbb{R}^{2}$ will be denoted by $|\mathbf{x}|$.

It is useful to introduce a (non-strictly) increasing, unbounded, integer sequence $([5])$

$$
n(t)=4 \sum_{s=1}^{t} U(s)=\frac{6}{\pi} t+\mathcal{O}(\sqrt{t}) .
$$

A convex lattice polygon $P$ has at most four edges with the same slope (where the edge slope is as defined previously). Therefore, for any integer $s, P$ has at most $4 U(s)$ edges with Euclidean length $\sqrt{s}$, if $x(e) \perp y(e)$ is assumed for any edge $e$. Consequently, a lower bound for the Euclidean perimeter of a convex lattice $n$-gon can be obtained by taking:

- $4 U(j)$ edges with length $\sqrt{j}(1 \leq j \leq t-1)$,

- $n-n(t-1)=n-4 \sum_{s=1}^{t-1} U(s)$ edges with length $\sqrt{t}$.

Since the above lower bound is established in a "greedy manner" it will be called the greedy lower bound and will be denoted by $\operatorname{glb}(n)$. Precisely,

$$
\operatorname{glb}(n)=(n-n(t-1)) \sqrt{t}+4 \sum_{s=1}^{t-1} \sqrt{s} U(s)
$$

where $t$ is uniquely determined by $n(t-1) \leq n<n(t)$.

Trivially, $\operatorname{glb}(n) \leq p(n)=\operatorname{per}\left(P_{n}\right)$, and if for $n=n(t)$ the equality $\operatorname{glb}(n)=p(n)$ can be reached only by the above described manner, this implies the uniqueness of $P_{n(t)}$, for any integer $t$. 
Let $A_{0}, A_{1}, \ldots, A_{n}=A_{0}$ be the counterclockwise ordered vertices of a convex lattice $n$-gon $P$, and $e_{1}=\left[A_{0} A_{1}\right], e_{2}=\left[A_{1} A_{2}\right], \ldots, e_{n}=\left[A_{n-1} A_{n}\right]$ be the vectors determined by consecutive vertices of $P$. Then $e_{1}, e_{2}, \ldots, e_{n}$ can be arranged into: south-east, north-east, north-west and south-west arc, in a natural way. Only the vertices of the south-east arc will be considered throughout the rest of the paper. Extension of the results to the other arcs is straightforward. The south-east arc vertices are $A_{0}, A_{1}, \ldots, A_{k}$, if $A_{0}$ is chosen from the vertices having the minimum $y$-coordinate, such that it has the minimum $x$-coordinate (the "left lowest" point) while the vertex $A_{k}$ is one of the vertices having the maximum $x$-coordinate, which has the minimum $y$-coordinate (the "lowest outermost right" point). For convenience and without loss of generality, we will assume $A_{0}=(0,0)$.

In the rest of the paper $(x(n, \alpha), y(n, \alpha))$ will denote the endpoint of the south-east arc edge of $P_{n}$ having slope $\alpha$, while $(\widetilde{x}(n, \alpha), \widetilde{y}(n, \alpha))$ is the corresponding vertex of the normalized polygon $P_{n}^{\prime}$.

3. Limit shape of the sequence $P_{n(t)}, t=1,2, \ldots$ In this section we derive the limit shape theorem for the sequence $P_{n(t)}^{\prime}$ of the normalized optimal convex lattice polygons which are introduced by Jarník [5]. This is the crucial case. Extension of the result to an arbitrary sequence $P_{n}^{\prime}$ follows easily from the fact that the number of edges of $P_{n}$ which are longer than $\sqrt{t}$ is $\mathcal{O}(\sqrt{t} \log t)$ where $t$ is determined by $n(t-1) \leq n<n(t)$.

We start with the asymptotic behavior of $x(n(t), \alpha)$ and $y(n(t), \alpha)$.

Lemma 3.1. Let $(x(n(t), \alpha), y(n(t), \alpha))$ be the endpoint of the edge of the south-east arc of $P_{n(t)}$ with slope $\alpha$. If $A_{0}$ is the origin then

$$
\begin{aligned}
& x(n(t), \alpha)=\frac{2 t \sqrt{t} \alpha}{\pi^{2} \sqrt{\alpha^{2}+1}}+\mathcal{O}(t \log t), \\
& y(n(t), \alpha)=\frac{2 t \sqrt{t}}{\pi^{2}}\left(1-\frac{1}{\sqrt{\alpha^{2}+1}}\right)+\mathcal{O}(t \log t) .
\end{aligned}
$$

Proof. The set of the slopes of the edges of the south-east arc of $P_{n(t)}$ coincides with the set $S(t)=\left\{p / q: p^{2}+q^{2} \leq t, p \perp q\right\}$. Also, the edges are arranged in the increasing order of their slopes, since $P_{n(t)}$ is convex. If $A_{i}=(x(n(t), \alpha), y(n(t), \alpha)), i=1,2, \ldots, n(t) / 4$, is a vertex of the south-east arc of $P_{n(t)}$ and $\alpha$ is the slope of $e_{i}=\left[A_{i-1} A_{i}\right]$, then

$$
x(n(t), \alpha)=\sum_{\substack{p \perp q \\ p^{2}+q^{2} \leq t \\ p / q \leq \alpha}} q \text { and } y(n(t), \alpha)=\sum_{\substack{p \perp q \\ p^{2}+q^{2} \leq t \\ p / q \leq \alpha}} p .
$$


Let us estimate $x(n(t), \alpha)$. The cardinality of the lattice point set $\mathcal{N}(v)=\left\{(q, p, r) \in \mathbb{Z}^{3}: q^{2}+p^{2} \leq v, q>0, p>0, p \perp q, \frac{p}{q} \leq \alpha, q \frac{\sqrt{t}}{\sqrt{v}} \geq r\right\}$ will be denoted by $N(v)$. An important remark is $N(t)=x(n(t), \alpha)$.

Next, let $B(v)$ denote the number of lattice points belonging to the $3 D$ body

$$
\mathcal{B}(v)=\left\{(x, y, z) \in \mathbb{R}^{3}: x^{2}+y^{2} \leq v, x>0, y>0, \frac{y}{x} \leq \alpha, x \frac{\sqrt{t}}{\sqrt{v}} \geq z\right\} .
$$

Due to Davenport's result ([4]), for arbitrary $t \geq 0, v \geq 0$, and $\alpha \in[0,2 \pi]$, we have

$$
\begin{aligned}
& |B(v)-\operatorname{volume}(\mathcal{B}(v))| \\
& \quad \leq 1+\sqrt{v}+\sqrt{\frac{\alpha^{2} v}{1+\alpha^{2}}}+\sqrt{t}+\frac{\sqrt{v t}}{2}+\frac{\alpha v}{2}+\sqrt{\frac{\alpha^{2} v t}{1+\alpha^{2}}} .
\end{aligned}
$$

Since

$$
\operatorname{volume}(\mathcal{B}(v))=\frac{v \sqrt{t} \alpha}{3 \sqrt{\alpha^{2}+1}},
$$

by setting $v=t / n^{2}$ we have (from (3)) the following estimate:

$$
B\left(\frac{t}{n^{2}}\right)=\frac{t \sqrt{t} \alpha}{3 n^{2} \sqrt{\alpha^{2}+1}}+\mathcal{O}\left(\frac{t}{n}\right)
$$

for $n=1, \ldots,\lfloor\sqrt{t}\rfloor$.

Further, for any $(a, b, c)$ belonging to $\mathcal{B}(v) \cap \mathbb{Z}^{3}$ the integer $j$ satisfying $(a, b, c) \in j^{2} \cdot \mathcal{N}\left(v / j^{2}\right)$ exists uniquely (precisely $\left.j=\operatorname{gcd}(a, b)\right)$. So, we conclude that $\mathcal{B}(v) \cap \mathbb{Z}^{3}$ equals the union of the nonoverlaping sets $i^{2} \cdot \mathcal{N}\left(v / i^{2}\right)$ for $i=1, \ldots,\lfloor\sqrt{v}\rfloor$. In other words

$$
\mathcal{B}(v) \cap \mathbb{Z}^{3}=\mathcal{N}(v) \cup 2^{2} \cdot \mathcal{N}\left(\frac{v}{2^{2}}\right) \cup \ldots \cup\lfloor\sqrt{t}\rfloor^{2} \cdot \mathcal{N}\left(\frac{v}{\lfloor\sqrt{t}\rfloor^{2}}\right),
$$

with $i \neq j \Rightarrow i^{2} \cdot \mathcal{N}\left(v / i^{2}\right) \cap j^{2} \cdot \mathcal{N}\left(v / j^{2}\right)=\emptyset$.

Consequently,

$$
B(v)=N(v)+N\left(\frac{v}{2^{2}}\right)+N\left(\frac{v}{3^{2}}\right)+\ldots=\sum_{i=1}^{\lfloor\sqrt{v}\rfloor} N\left(\frac{v}{i^{2}}\right)
$$

because $N\left(v / i^{2}\right)=0$ for $i=\lfloor\sqrt{v}\rfloor+1,\lfloor\sqrt{v}\rfloor+2, \ldots$ Analogously, for $v=$ $t / n^{2}$,

$$
B\left(\frac{t}{n^{2}}\right)=\sum_{m=1}^{\infty} N\left(\frac{t}{n^{2} m^{2}}\right)=\sum_{m=1}^{\left\lfloor\sqrt{t / n^{2}}\right\rfloor} N\left(\frac{t}{n^{2} m^{2}}\right) .
$$


Finally, by a standard technique we have

$$
\begin{aligned}
x(n(t), \alpha) & =N(t) \\
& =\sum_{l=1}^{\lfloor\sqrt{t}\rfloor} N\left(\frac{t}{l^{2}}\right)\left(\sum_{n \mid l} \mu(n)\right)=\sum_{n=1}^{\lfloor\sqrt{t}\rfloor}\left(\mu(n) \sum_{m=1}^{\left\lfloor\sqrt{t / n^{2}}\right\rfloor} N\left(\frac{t}{n^{2} m^{2}}\right)\right) \\
& =\sum_{n=1}^{\lfloor\sqrt{t}\rfloor} \mu(n) B\left(\frac{t}{n^{2}}\right)=\sum_{n=1}^{\lfloor\sqrt{t}\rfloor} \mu(n)\left(\frac{t \sqrt{t} \alpha}{3 n^{2} \sqrt{\alpha^{2}+1}}+\mathcal{O}\left(\frac{t}{n}\right)\right) \\
& =\frac{t \sqrt{t} \alpha}{3 \sqrt{\alpha^{2}+1}} \sum_{n=1}^{\lfloor\sqrt{t}\rfloor} \frac{\mu(n)}{n^{2}}+\mathcal{O}\left(t \sum_{n=1}^{\sqrt{t}} \frac{1}{n}\right) \\
& =\frac{t \sqrt{t} \alpha}{3 \sqrt{\alpha^{2}+1}}\left(\sum_{n=1}^{\infty} \frac{\mu(n)}{n^{2}}-\sum_{n=\lfloor\sqrt{t}\rfloor+1}^{\infty} \frac{\mu(n)}{n^{2}}\right)+\mathcal{O}\left(t \int_{1}^{\sqrt{t}} \frac{d x}{x}\right) \\
& =\frac{t \sqrt{t} \alpha}{3 \sqrt{\alpha^{2}+1}} \cdot \frac{1}{\zeta(2)}+\mathcal{O}(t \log t)=\frac{2 t \sqrt{t} \alpha}{\pi^{2} \sqrt{\alpha^{2}+1}}+\mathcal{O}(t \log t) .
\end{aligned}
$$

The asymptotic behavior of $y(n(t), \alpha)$ can be derived analogously-just notice that the analogue for $N(v)$, say $\widetilde{N}(v)$, is the number of lattice points $(q, p, r)$ satisfying $q^{2}+p^{2} \leq v, p, q>0, p \perp q, p / q \leq \alpha, p \sqrt{t} / \sqrt{v} \geq r$.

Now, we can prove the required limit shape theorem which shows that the sequence of normalized optimal polygons $P_{n(t)}^{\prime}$ tends to a circle as $t \rightarrow \infty$ (or equivalently, as $n(t) \rightarrow \infty$ ).

THEOREM 3.1. There is a positive constant $c_{1}$ such that the suitable translate $P_{n(t)}^{\prime}$ is contained in the ring of points $\mathbf{x}$ with

$$
|| \mathbf{x}\left|-\frac{1}{2}\right|<c_{1} t^{-1 / 2} \log t
$$

for t large enough.

Proof. This is a direct consequence of the previous lemma. Noticing that $\Delta\left(P_{n(t)}\right)$ equals both $2 x(n(t), \alpha)$ and $2 y(n(t), \alpha)$, for $\alpha=\infty$, we have

$$
\Delta(P(n(t)))=\frac{4 t \sqrt{t}}{\pi^{2}}+\mathcal{O}(\log t) .
$$

Further, if $A_{0}=(0,0)$ is assumed and $P_{n(t)}^{\prime}$ is translated by the vector $(0,-1 / 2)$, then the vertices $(\widetilde{x}(n(t), \alpha), \widetilde{y}(n(t), \alpha))$ of $P_{n(t)}^{\prime}$ satisfy

$$
\begin{aligned}
& \widetilde{x}(n(t), \alpha)=\frac{\alpha}{2 \sqrt{\alpha^{2}+1}}+\mathcal{O}\left(t^{-1 / 2} \log t\right), \\
& \widetilde{y}(n(t), \alpha)=\frac{-1}{2 \sqrt{\alpha^{2}+1}}+\mathcal{O}\left(t^{-1 / 2} \log t\right) .
\end{aligned}
$$


Consequently, ||$(\widetilde{x}(n(t), \alpha), \widetilde{y}(n(t), \alpha))\left|-\frac{1}{2}\right|<c_{1} t^{-1 / 2} \log t$ for some constant $c_{1}>0$ and $t$ large enough. Since

$$
\frac{|e|}{\Delta\left(P_{n(t)}\right)} \leq \frac{\sqrt{t}}{\Delta\left(P_{n(t)}\right)}=\mathcal{O}\left(t^{-1}\right)
$$

for any edge $e$ of $P_{n(t)}$, we conclude that all edges of $P_{n(t)}^{\prime}$ belong to the same ring, as well.

\section{References}

[1] T. M. Apostol, Introduction to Analytic Number Theory, Springer, New York, 1976.

[2] I. Bárány, Affine perimeter and limit shape, J. Reine Angew. Math. 484 (1997), 71-84.

[3] E. Bombieri and J. Pila, The number of integral points on arcs and ovals, Duke Math. J. 59 (1989), 337-357.

[4] H. Davenport, On a principle of Lipschitz, J. London Math. Soc. 26 (1951), 179-183.

[5] V. Jarník, Über die Gitterpunkte auf konvexen Kurven, Math. Z. 24 (1926), 500-518.

[6] W. M. Schmidt, Integer points on curves and surfaces, Monatsh. Math. 99 (1985), $45-72$.

[7] Ya. G. Sinai, A probabilistic approach to the analysis of the statistics of convex polygonal lines, Funktsional. Anal. i Prilozhen. 28 (1994), no. 2, 41-48 (in Russian).

[8] H. P. F. Swinnerton-Dyer, The number of lattice points on a convex curve, J. Number Theory 6 (1974), 128-135.

[9] A. M. Vershik, The limit shape of convex lattice polygons and related topics, Funktsional. Anal. i Prilozhen. 28 (1994), no. 1, 16-25 (in Russian).

Cardiff University

Computer Science

Queen's Buildings, Newport Road

P.O. Box 916

Cardiff CF24 3XF

Wales, U.K.

E-mail: J.Zunic@cs.cf.ac.uk
Mathematical Institute Serbian Academy of Science and Arts

Belgrade, Serbia

Received on 28.8.2001

and in revised form on 24.4.2002 